\title{
Conjugated linoleic acid reduces permeability and fluidity of adipose plasma membranes from obese Zucker rats
}

\author{
Ana P. Martins ${ }^{a}$, Paula A. Lopes ${ }^{b}$, Susana V. Martins ${ }^{b}$, Ana Madeira ${ }^{a, c}$, Nuno C. Santos ${ }^{\text {d }}$, José A.M. Prates ${ }^{\text {b }}$, \\ Teresa F. Moura ${ }^{a}$, Graça Soveral ${ }^{\mathrm{a}, \mathrm{e}, *}$ \\ ${ }^{a}$ REQUIMTE, Dep. Química, FCT-UNL, 2829-516 Caparica, Portugal \\ ${ }^{\mathrm{b}}$ CIISA, Faculdade de Medicina Veterinária, TULisbon, 1300-477 Lisboa, Portugal \\ ${ }^{\mathrm{C}}$ Institute for Research in Biomedicine (IRB, Barcelona), C/Baldiri Reixac 10, Barcelona, Spain \\ ${ }^{\mathrm{d}}$ Instituto de Medicina Molecular, Faculdade de Medicina, Universidade de Lisboa, 1649-028 Lisboa, Portugal \\ e Faculdade de Farmácia, Universidade de Lisboa, 1649-003 Lisboa, Portugal
}

\section{A R T I C L E I N F O}

Article history:

Received 10 June 2010

Available online $\mathrm{xxxx}$

\section{Keywords:}

Conjugated linoleic acid

Adipose plasma membrane

Glycerol permeability

Water permeability

Membrane fluidity

Obese Zucker rats

\begin{abstract}
Conjugated linoleic acid (CLA) is a dietary fatty acid frequently used as a body fat reducing agent whose effects upon cell membranes and cellular function remain unknown. Obese Zucker rats were fed atherogenic diets containing saturated fats of vegetable or animal origin with or without $1 \%$ CLA, as a mixture of $c i s(c) 9$,trans $(t) 11$ and $t 10, c 12$ isomers. Plasma membrane vesicles obtained from visceral adipose tissue were used to assess the effectiveness of dietary fat and CLA membrane incorporation and its outcome on fluidity and permeability to water and glycerol. A significant decrease in adipose membrane fluidity was correlated with the changes observed in permeability, which seem to be caused by the incorporation of the $t 10, c 12$ CLA isomer into membrane phospholipids. These results indicate that CLA supplementation in obese Zucker rats fed saturated and cholesterol rich diets reduces the fluidity and permeability of adipose membranes, therefore not supporting CLA as a body fat reducing agent through membrane fluidification in obese fat consumers.
\end{abstract}

(c) 2010 Published by Elsevier Inc.

\section{Introduction}

Increased consumption of highly energetic food with high levels of saturated fats have led to a threefold increase of obesity rates in industrialized societies contributing to human premature morbidity and mortality [1]. Facing this worldwide health problem, new compounds that might prevent or even reverse such scenario have attracted scientific attention. One of the natural compounds most studied in the last decades as a body fat reducing agent is the conjugated linoleic acid (CLA). CLA is a generic term for a mixture of geometrical and positional isomers of linoleic acid $(18: 2 n-6)$ with conjugated double bonds in either cis $(c)$ or trans $(t)$ configuration, found mainly in ruminant-derived foods [2]. Being a dietary fatty acid, CLA reveals an impressive range of promising health benefits including reduction of body fat mass [2] but the mechanisms by which CLA elicits its promising effects remain unknown. It is known that CLA isomers rapidly incorporate into cell membrane lipids $[3,4]$, but their outcome on cell membranes and changes in cellular function is still unexploited. Due to the potential anti-adipogenic effect attributed to CLA, it would be interesting

\footnotetext{
* Corresponding author at: REQUIMTE, Dep. Química, FCT-UNL, 2829-516 Caparica, Portugal. Fax: +351 212948550

E-mail addresses: gsoveral@ff.ul.pt, soveral@dq.fct.unl.pt (G. Soveral).
}

to gain insights on its effect on adipose tissue and specifically on adipose membrane composition, permeability and fluidity. It has been reported that incorporation of polyunsaturated fatty acids (PUFA) into cellular membranes increases fluidity and permeability [5]. Moreover, the effect of diet supplementation with CLA has been shown to modify lipid composition and glycerol permeability of rat kidney membranes [4]. Glycerol is a key substrate involved in lipogenesis and lipolysis of adipose tissue; glycerol release and uptake from the adipocyte is mediated by aquaporin 7 (AQP7), a glycerol-channel that regulates glycerol accumulation [6].

The present study aimed at assessing the effect of dietary CLA on adipose membrane lipid composition and further correlate it with changes in permeability and fluidity. In order to reproduce the most frequent human group consuming CLA as a promoter of body fat mass reduction, our experimental design included obese Zucker rats fed atherogenic diets containing saturated fats of vegetable and animal origins (palm oil and ovine fat) and with or without 1\% CLA mixture. An enriched preparation of plasma membrane vesicles, osmotically responsive and suitable for transport experiments, has been obtained from rat white adipose tissue. Membrane vesicles were analyzed regarding their total lipid profile and CLA incorporation into membrane phospholipids. Permeabilities to glycerol and water were assessed and used to estimate the activation energy of transport, a valuable parameter indicating 
the relative contribution of specific transport proteins for membrane permeation. The relationship between membrane composition and fluidity was further characterized by fluorescence anisotropy, a method sensitive to the packing density induced by cholesterol and membrane lipids incorporation.

\section{Materials and methods}

\subsection{Experimental design: animals and diets}

The experimental protocol was reviewed by the Ethics Commission of CIISA/FMV and approved by the Animal Care Committee of the National Veterinary Authority following European Union guidelines (N. 86/609/EEC).

Male obese Zucker $(\mathrm{fa} / \mathrm{fa})$ rats $(n=32)$ were obtained from Harlan Interfauna Iberia (Barcelona, Spain), aging 5 weeks old. Animals were housed individually and after 1 week of adaptation, rats were fed semi-purified atherogenic diets (Provimi Kliba, SA, Switzerland), with $2 \%(\mathrm{w} / \mathrm{w})$ cholesterol (plus $0.5 \%(\mathrm{w} / \mathrm{w})$ sodium cholate to improve cholesterol absorption) and $15 \%(\mathrm{w} / \mathrm{w})$ of fat with distinct fatty acid composition [7]. Rats were divided into four groups with eight animals each, according to the dietary fat: group $\mathrm{P}-11.3 \%$ palm oil $(\mathrm{w} / \mathrm{w})$ plus $3.8 \%(\mathrm{w} / \mathrm{w})$ sunflower oil; group PCLA - $11.3 \%(\mathrm{w} / \mathrm{w})$ palm oil plus $2.5 \%$ sunflower oil $(\mathrm{w} / \mathrm{w})$ plus $1.2 \%(\mathrm{w} / \mathrm{w})$ CLA; group $\mathrm{O}-11.3 \%(\mathrm{w} / \mathrm{w})$ ovine fat plus $3.8 \%(\mathrm{w} /$ w) sunflower oil; group OCLA - $11.3 \%(\mathrm{w} / \mathrm{w})$ ovine fat plus $2.5 \%$ $(\mathrm{w} / \mathrm{w})$ sunflower oil plus $1.2 \%(\mathrm{w} / \mathrm{w})$ CLA. The CLA oil contained similar proportions of $c 9, t 11$ and $t 10, c 12$ isomers with a purity level of $80 \%$ (PharmaNutrients Inc., USA). After 14 weeks of trial, rats were fasted for $12 \mathrm{~h}$ and sacrificed by decapitation under light isofluorane anaesthesia (Abbott, USA). The epididymal fat pads were removed, washed with saline, weighted and stored at $-80^{\circ} \mathrm{C}$ for subsequent analysis.

\subsection{Preparation of membrane vesicles from epididymal fat}

Membrane vesicles were prepared from rat's epididymal fat by differential centrifugation with buffer without detergents. Approximately $4 \mathrm{~g}$ of epididymal white fat tissue from each rat was chopped into small pieces, removing visible endothelial tissue, and homogenized in mannitol-Hepes buffer (100 mM mannitol, $10 \mathrm{mM}$ Tris-Hepes, $\mathrm{pH}$ 7.4) in a warring blender for $2 \mathrm{~min}$. The homogenate was filtered through a $70 \mu$ m nylon mesh to further separate the vascular stroma from fat. The filtrate was centrifuged at $1800 \mathrm{~g}$ for $10 \mathrm{~min}$ and the supernatant, consisting in intracellular fat, was discarded. The infranatant was centrifuged at $46,000 \mathrm{~g}$ for $45 \mathrm{~min}$ at $10^{\circ} \mathrm{C}$ to obtain a pellet of crude membranes, and further washed in the same buffer. The membrane pellet was resuspended in mannitol-Hepes buffer, transferred to a syringe and sheared by vigorously passing it 10 times through a 21-gauge needle and immediately used for transport experiments. Protein content was determined by the Bradford technique [8].

\subsection{Vesicle size determination}

Vesicle size of all the membrane preparations was determined by the Quasi-Elastic Light Scattering (QELS) technique (Brookhaven Instruments BI-90).

\subsection{Immunoblot analysis}

Proteins were resolved in $12.5 \%(\mathrm{w} / \mathrm{v})$ SDS-PAGE and transferred to PVDF membrane (Immobilon-P membrane; Millipore). After blocking with $10 \%$ nonfat powder milk in PBS for $1 \mathrm{~h}$ at room temperature, the membrane was incubated overnight at $4{ }^{\circ} \mathrm{C}$ with primary rabbit antibodies against GLUT4 (BD Transduction Laboratories, Canada), caveolin (Dept. Bioquímica i Biologia Molecular, Facultat de Biologia, Universitat de Barcelona, Spain) and AQP7 (Millipore). Immunoblot analysis was performed with the enhanced chemiluminescence system and horseradish peroxidase conjugated with anti-rabbit IgG (Amersham).

\subsection{Fatty acid composition and cholesterol content of adipose membranes}

After membrane vesicle lyophilisation, fatty acids were converted to methyl esters (FAME) according to [9]. The resulting FAME were analyzed by gas chromatography (GC), using a capillary column (Omegawax 250; Supelco, USA), equipped with a flameionization detector. The fatty acid composition was expressed as $\mathrm{g} / 100 \mathrm{~g}$ of total fatty acids identified.

Total cholesterol was extracted from lyophilised membrane vesicles through a direct saponification with saturated methanolic $\mathrm{KOH}$ solution [10]. Cholesterol was separated and identified using a high performance liquid chromatography (HPLC) equipment (Agilent 1100 Series, Agilent Technologies Inc., USA) by normal phase (Zorbax Rx-Sil column, Agilent Technologies Inc.). Total cholesterol content was calculated, in triplicate, based on the external standard technique, from a standard curve for peak area versus cholesterol concentration and expressed as $\mathrm{mg} / \mathrm{g}$ vesicles.

\subsection{Stopped-flow experiments on water and glycerol permeability}

Stopped-flow experiments were performed on a HI-TECH Scientific PQ/SF-53 apparatus, which has a 2 ms dead time, temperature controlled and interfaced with a PC microcomputer. Experiments were performed at temperatures from $14^{\circ} \mathrm{C}$ to $37^{\circ} \mathrm{C}$. Five runs were usually stored and analysed in each experimental condition. For the measurement of osmotic water permeability, membrane vesicles $(0.2 \mathrm{mg}$ protein $/ \mathrm{ml}$ ) resuspended in mannitol-Hepes buffer (120 mOsM) were mixed with an equal amount of isosmotic or hyperosmotic (240 mOsM) mannitol solutions to reach an inwardly directed gradient of the impermeant solute. The kinetics of vesicle shrinkage was measured from the time course of scattered light intensity at $400 \mathrm{~nm}$ until a stable light scatter signal was attained. The osmotic water permeability coefficient $\left(P_{f}\right)$ was estimated by fitting the light scatter signal to a single exponential curve and using the linear relation between $P_{f}$ and the exponential time constant $k$ [11], $P_{f}=k\left(V_{o} / A\right)\left(1 / V_{w}\left(\text { osm }_{\text {out }}\right)_{\infty}\right)$, where $V_{w}$ is the molar volume of water, $V_{0} / A$ is the initial volume to area ratio of the vesicle preparation, and $\left(o s m_{\text {out }}\right)_{\infty}$ is the final medium osmolarity after the applied osmotic gradient. For glycerol permeability, membrane vesicles equilibrated in 120 mOsM mannitol-Hepes buffer were confronted to an external solution where the impermeant solute was partially replaced by glycerol (60 mOsM mannitol plus 180 mOsM glycerol, creating an inwardly directed glycerol gradient). After the first fast vesicle shrinkage due to water outflow, glycerol influx in response to its chemical gradient was followed by water influx with subsequent vesicle reswelling. Glycerol permeability was calculated as $P_{g l y}=k\left(V_{o} / A\right)$, where $k$ is the single exponential time constant fitted to the light scattering signal of glycerol influx [12]. For inhibitor assays, vesicles were incubated for $5 \mathrm{~min}$ with $0.5 \mathrm{mM} \mathrm{HgCl}_{2}$ immediately prior to stopped flow injection. All solution osmolarities were determined from freezing point depression on a semi-micro osmometer (Knauer GmbH, Germany) using standards of 100 and 400 mOsM.

The activation energy $E_{a}$ of water and glycerol transport was calculated from the slope of the Arrhenius plot $\left(\ln P_{f}\right.$ or $\ln P_{\text {gly }}$ as a function of $1 / T$ ) multiplied by the gas constant $R$. 


\subsection{Membrane fluidity measurements}

Membrane fluidity was studied by a fluorescence polarization method, which measures the fluorescence anisotropy; of two probes incorporated in the membrane: 1,6-diphenyl-1,3,5hexatriene (DPH), or 1-(4-(trimethylamino)-phenyl)-6-phenyl1,3,5-hexatriene (TMA-DPH), (Molecular Probes, USA). DPH is incorporated inside the membrane, at the fatty acyl group's level, while TMA-DPH is anchored by its cationic part at the membrane/water interface, probing the membrane region closer to the phospholipids head groups [13]. DPH $1 \mathrm{mM}$ in acetone or TMA-DPH $0.5 \mathrm{mM}$ in dimetylformamide were diluted $1: 180$ in mannitol-Hepes buffer, vigorously stirred for $1 \mathrm{~min}$, mixed with an aliquot of the vesicle suspension (final protein $20 \mu \mathrm{g} / \mathrm{ml}$ ) and incubated $30 \mathrm{~min}$ in the dark, at $37^{\circ} \mathrm{C}$ for DPH and at room temperature for TMA-DPH. Blank samples were prepared replacing the probes by identical volumes of their solvents. As the fluorescent probes reach equilibrium between the aqueous and lipid phases and the unincorporated probes almost do not fluoresce [14], no washing was needed. Membrane fluidity was assessed by fluorescence anisotropy $(r)$ defined by the equation $r=\left(I_{V V}-G I_{V H}\right) /$ $\left(I_{V V}+2 G I_{V H}\right)$, where $I_{V V}$ and $I_{V H}$ are the fluorescence intensities and the subscripts indicate the vertical $(V)$ or horizontal $(H)$ orientations of the excitation and emission polarizers, and $G=I_{H V} / I_{H H}$ is the instrumental factor [15]. DPH fluorescence was measured at an excitation wavelength ( $\left.\lambda_{\text {exc }}\right)$ of $357 \mathrm{~nm}$ and an emission wavelength $\left(\lambda_{\mathrm{em}}\right)$ of $428 \mathrm{~nm}$. For TMA-DPH, $\lambda_{\mathrm{exc}}=343 \mathrm{~nm}$ and $\lambda_{\mathrm{em}}=427 \mathrm{~nm}$. The fluorescence intensity data used for calculations were the average of three identical aliquots (after blank subtraction) measured on a Varian Cary Eclipse fluorescence spectrophotometer (Mulgrave, Australia).

\subsection{Statistics}

Statistical analysis was performed using the statistical analysis system (SAS) software package, v9.1 (SAS Institute, USA). Data were mean and standard error of the mean (SEM). The procGLM procedure was used to perform a $2 \times 2$ factorial analysis to determine significant effects of CLA, fat origin and their respective interaction $(\mathrm{CLA} \times \mathrm{fat})$. In the case of interaction, significant differences between groups were identified using Tukey's post-hoc test at $P<0.05$.

\section{Results}

\subsection{Characterization of membrane vesicles from epididymal fat}

Vesicle size of membrane preparations within each dietary treatment $(n=8)$ revealed homogeneous populations with monomodal distributions, showing a mean diameter of (303 \pm 42 , $297 \pm 54,319 \pm 50$ and $276 \pm 44) \mathrm{nm}$ for P, PCLA, O and OCLA groups, respectively.

Western blots of aliquots from the initial fat tissue homogenate and from the final adipose membrane preparations were incubated with antibody anti-GLUT4 and anti-caveolin. Additionally, membrane preparations were incubated with anti-AQP7. An intense band of GLUT4, the insulin-stimulated glucose transporter [16], was depicted in the membrane vesicles but was almost absent in the homogenate fraction (Fig. 1). Likewise, the band for caveolin, a constitutive protein of plasma membranes of mature adipocytes [16], was stronger in the final vesicle preparation. AQP7 was also detected in the adipose membranes, despite in a fainter band than caveolin. Altogether, these results assure purified and homogeneous adipose plasma membrane preparations.
A

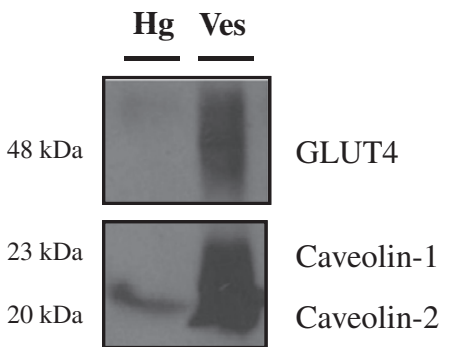

B

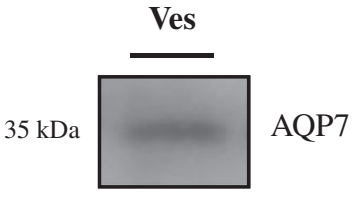

Fig. 1. (A) GLUT4 and caveolin proteins in the initial fat tissue homogenate ( $\mathrm{Hg}$, lane 1$)$ and in the final adipose membrane vesicles (Ves, lane 2) (10 $\mu$ g protein). (B) AQP7 in the final adipose membrane vesicles (Ves, $50 \mu \mathrm{g}$ protein).

\subsection{Fatty acid membrane composition depends on dietary fat and CLA supplementation}

The fatty acid composition of membrane vesicles from the four dietary treatments is shown in Table 1 . Total cholesterol content was higher in membranes from palm oil groups and not affected by CLA supplementation. The deposition of total saturated fatty acids (SFA) was not different among groups. However, while the 16:0 incorporation was greater in palm oil groups, the 18:0 and 20:0 fatty acids were enhanced in ovine fat groups. The differences found for monounsaturated fatty acids (MUFA) were related to the dietary fat origin, being consistently higher in palm oil groups, with and without CLA. Yet, the incorporation of 18:1 fatty acid was affected by both CLA and fat origin effects in separate, being higher in ovine fat diets.

The sum of PUFA and specifically $n-6$ fatty acids, was not affected by CLA, fat origin or their respective interaction. However, the $n-3$ sum was influenced by the fat origin. Regarding the CLA isomeric profile, major differences were observed between the four dietary groups. The sum of CLA isomers increased by CLA mixture supplementation and was higher in diets based on animal fat. However, isomeric incorporation in adipose membranes was distinct. The $t 10, c 12$ isomer was detected only by CLA supplementation, while $c 9, t 11$ deposition was influenced by both CLA and fat origin and was significantly larger in both fat based diets.

\subsection{CLA decreases permeability and fluidity of adipose membrane vesicles}

For water transport experiments, vesicles in isotonic buffer were subjected to a hyperosmotic gradient with mannitol, as depicted in Fig. 2A. Fig. 2B shows a typical trace where a glycerol gradient was imposed. Fast vesicle shrinkage due to water outflow (first part of the trace) is followed by vesicle reswelling; as glycerol enters due to its chemical gradient, vesicles progressively swell until they reach equilibrium. The time courses of vesicle volume changes are used to calculate $P_{f}$ and $P_{g l y}$.

Fig. 3 shows the water and glycerol permeability coefficients obtained at $23{ }^{\circ} \mathrm{C}$ for the various membrane preparations (for each group, $n=8)$. Average $P_{f} \pm$ SEM were $(1.88 \pm 0.113,1.59 \pm 0.260$, $2.08 \pm 0.080$ and $1.75 \pm 0.080) \times 10^{-3} \mathrm{~cm} \mathrm{~s}^{-1}$ for P, PCLA, O and OCLA groups, respectively. For the same groups, $P_{\text {gly }} \pm$ SEM were $(4.77 \pm 0.274, \quad 4.16 \pm 0.275, \quad 5.22 \pm 0.183$ and $4.43 \pm 0.348) \times$ $10^{-7} \mathrm{~cm} \mathrm{~s}^{-1}$. A significant CLA effect was detected for both permeabilities as dietary groups with CLA presented lower values in relation to their counterparts $\left(P<0.01\right.$ for $P_{f}$ and $P<0.05$ for $\left.P_{g / y}\right)$.

The activation energy values $\left(E_{a}\right)$ for water and glycerol transport were similar among dietary groups, ranging from $24.4 \pm 0.36$ to $24.9 \pm 0.35 \mathrm{kcal} \mathrm{mol}^{-1}\left(102.1 \pm 1.51\right.$ to $\left.104.1 \pm 1.46 \mathrm{~kJ} \mathrm{~mol}^{-1}\right)$ 
Table 1

Cholesterol content (mg/g vesicles) and fatty acid (\% of total FAME) profile of membrane vesicles from the dietary treatments tested.

\begin{tabular}{|c|c|c|c|c|c|c|c|c|}
\hline & \multicolumn{5}{|c|}{ Dietary treatments } & \multicolumn{3}{|c|}{ Significance level } \\
\hline & $P$ & PCLA & 0 & OCLA & SEM & CLA & Fat & $\mathrm{CLA} \times$ Fat \\
\hline $\begin{array}{l}\text { Cholesterol content } \\
\text { Fatty acid profile }\end{array}$ & 0.044 & 0.040 & 0.027 & 0.036 & 0.004 & ns & $*$ & ns \\
\hline $14: 0$ & $0.867^{\mathrm{b}}$ & $1.05^{\mathrm{a}}$ & $1.02^{\mathrm{a}}$ & $1.07^{\mathrm{a}}$ & 0.033 & $* *$ & * & * \\
\hline $15: 0$ & 0.114 & 0.140 & 0.237 & 0.247 & 0.010 & ns & ${ }^{* * *}$ & ns \\
\hline $16: 0$ & 23.6 & 24.1 & 18.7 & 18.8 & 0.380 & ns & ${ }^{* * *}$ & ns \\
\hline $16: 1$ & 0.564 & 0.657 & 0.693 & 0.698 & 0.027 & ns & ${ }^{* *}$ & ns \\
\hline $16: 1 c 9$ & 4.39 & 4.81 & 4.05 & 3.81 & 0.197 & ns & ${ }^{* *}$ & ns \\
\hline $17: 0$ & 0.300 & 0.357 & 0.644 & 0.659 & 0.029 & ns & *** & ns \\
\hline $18: 0$ & $10.9^{\mathrm{b}}$ & $10.1^{\mathrm{b}}$ & $13.4^{\mathrm{a}}$ & $14.9^{\mathrm{a}}$ & 0.451 & ns & ${ }^{* * *}$ & ${ }^{*}$ \\
\hline $18: 1$ & 3.46 & 3.94 & 5.42 & 5.84 & 0.075 & & $* * *$ & ns \\
\hline $18: 1 c 9$ & 28.5 & 26.4 & 24.1 & 22.9 & 0.868 & ns & ${ }^{* *}$ & ns \\
\hline $18: 2 n-6$ & 11.4 & 11.9 & 12.2 & 12.1 & 0.247 & ns & ${ }^{*}$ & ns \\
\hline CLA-c9t11 & 0.273 & 1.15 & 1.59 & 2.35 & 0.063 & ${ }^{* * *}$ & ${ }^{* * *}$ & ns \\
\hline CLA- $t 10 c 12$ & n.d. & 0.369 & n.d. & 0.439 & 0.028 & $* * *$ & ns & ns \\
\hline$\Sigma$ CLA $t t$ & n.d. & 0.178 & 0.049 & 0.226 & 0.016 & $* * *$ & ${ }^{* *}$ & ns \\
\hline $18: 3 n-3$ & 0.113 & 0.109 & 0.343 & 0.294 & 0.022 & ns & $* * *$ & ns \\
\hline $20: 0$ & 0.293 & 0.305 & 0.409 & 0.376 & 0.040 & ns & ${ }^{*}$ & ns \\
\hline $20: 1 n-9$ & 0.233 & 0.187 & 0.146 & 0.109 & 0.007 & $* * *$ & ${ }^{* * *}$ & ns \\
\hline $20: 3 n-6$ & 0.536 & 0.508 & 0.597 & 0.498 & 0.037 & ns & ns & ns \\
\hline $20: 4 n-6$ & 6.84 & 7.12 & 7.71 & 7.09 & 0.693 & ns & ns & ns \\
\hline $20: 5 n-3$ & n.d. & 0.062 & 0.142 & 0.160 & 0.014 & $* *$ & ${ }^{* * *}$ & ns \\
\hline $21: 0$ & 0.672 & 0.730 & 0.953 & 0.916 & 0.117 & ns & ns & ns \\
\hline $23: 0$ & 0.344 & 0.143 & 0.187 & 0.157 & 0.055 & & ns & ns \\
\hline $22: 4 n-6$ & 0.930 & 0.848 & 0.811 & 0.621 & 0.100 & ns & ns & ns \\
\hline $22: 5 n-3$ & $0.096^{\mathrm{c}}$ & $0.109^{b c} b, c$ & $0.362^{\mathrm{a}}$ & $0.215^{\mathrm{b}}$ & 0.029 & & $* * *$ & ${ }^{* *}$ \\
\hline $22: 6 n-3$ & 0.119 & 0.073 & 0.208 & 0.180 & 0.024 & ns & ${ }^{* * *}$ & ns \\
\hline$\Sigma$ Unidentified & 5.51 & 4.74 & 5.99 & 5.42 & 0.397 & ns & ns & ns \\
\hline$\Sigma$ SFA & 37.0 & 36.9 & 35.5 & 37.1 & 0.424 & ns & ns & ns \\
\hline$\Sigma$ MUFA & 37.2 & 36.0 & 34.5 & 33.4 & 1.06 & ns & & ns \\
\hline$\Sigma$ PUFA & 20.0 & 20.7 & 22.4 & 21.1 & 0.911 & ns & ns & ns \\
\hline$\Sigma$ CLAs & 0.273 & 1.70 & 1.64 & 3.01 & 0.080 & $* * *$ & ${ }^{* * *}$ & ns \\
\hline$\sum n-3$ & 0.328 & 0.352 & 1.05 & 0.849 & 0.061 & ns & $* * *$ & ns \\
\hline$\sum n-6$ & 19.7 & 20.4 & 21.4 & 20.3 & 0.874 & ns & ns & ns \\
\hline
\end{tabular}

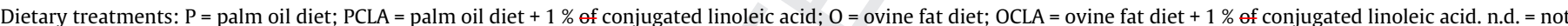

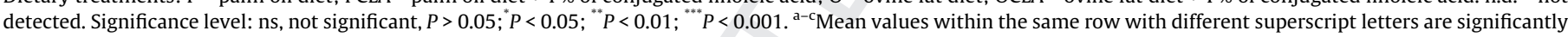

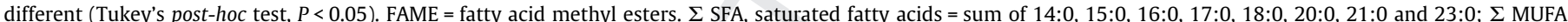

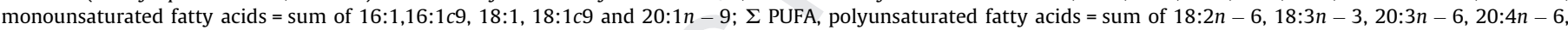

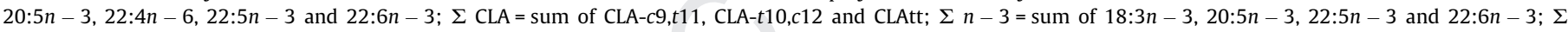
$n-6=$ sum of $18: 2 n-6,20: 3 n-6,20: 4 n-6$ and $22: 4 n-6$.

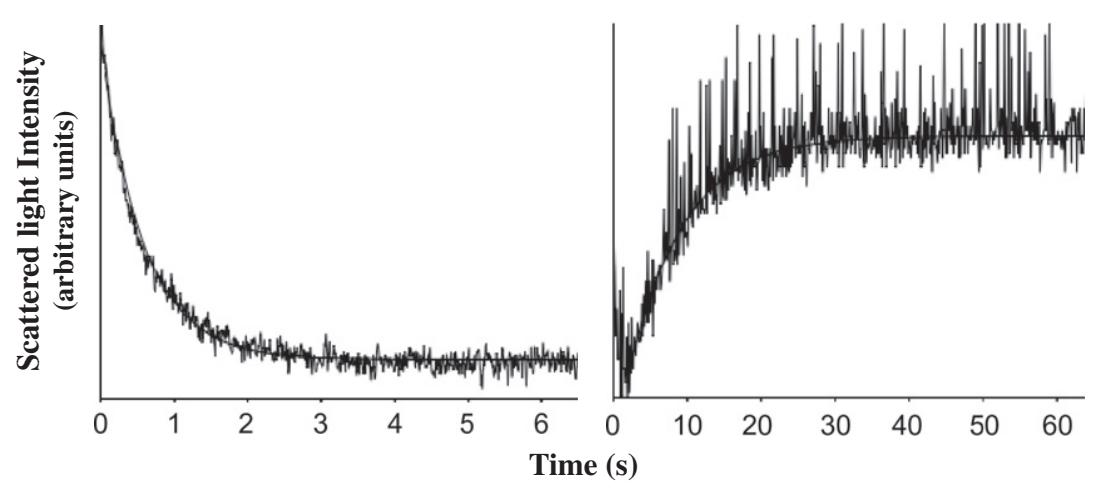

A
B 
A

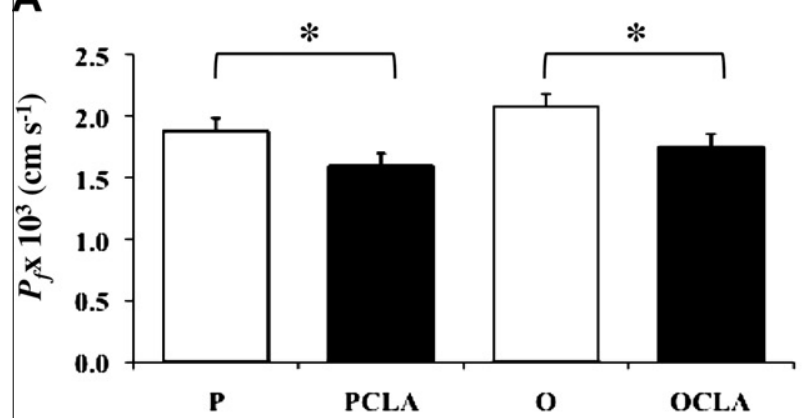

B

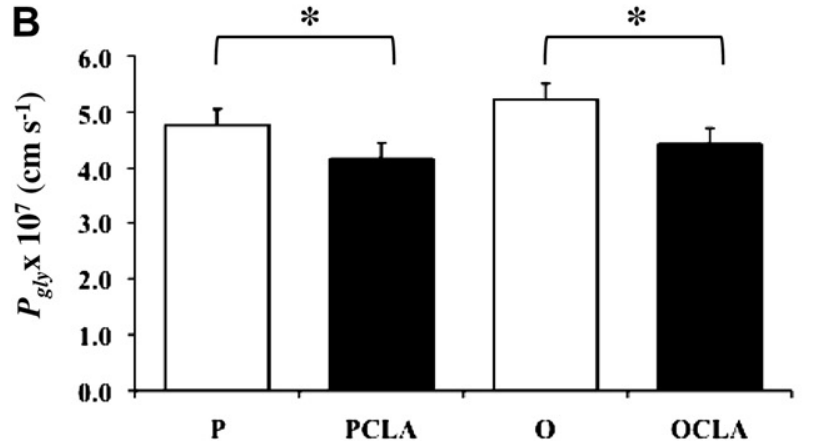

C

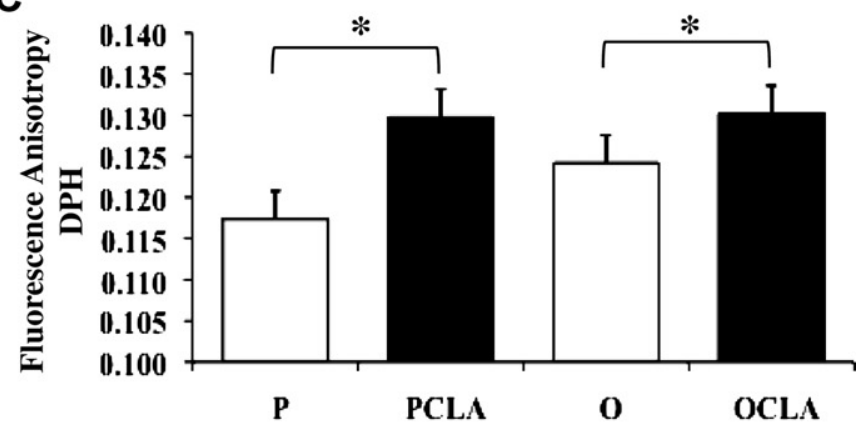

D

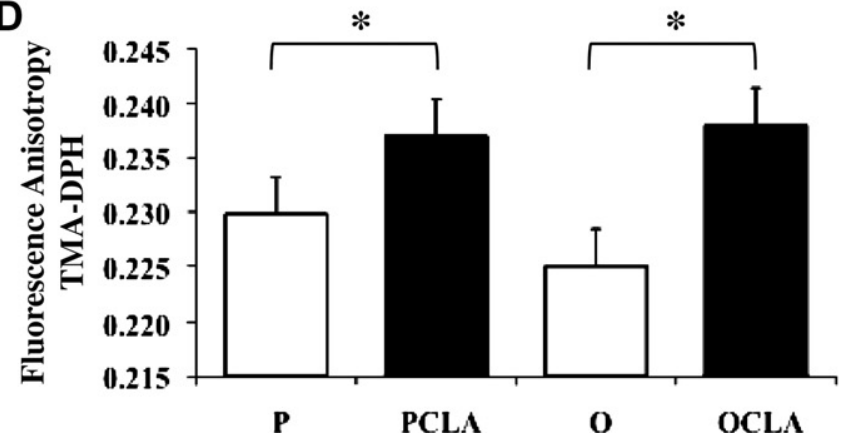

has been reported and correlated with the susceptibility to obesity of obese high fat consumers [17]. To confirm the lipid bilayer as the main pathway for water and glycerol permeation, adipose membrane vesicles were pre-incubated with $\mathrm{HgCl}_{2}$, a known inhibitor of aquaporin function [18], before permeability assays. Results of $P_{f}$ or $P_{g l y}$ did not change after $\mathrm{HgCl}_{2}$ treatment $(P \geqslant 0.05$; Fig. 4$)$, suggesting a non-channel mediated water and glycerol transport.

The relative changes in fluorescence anisotropy of DPH and TMA-DPH in membrane vesicles from each dietary treatment are

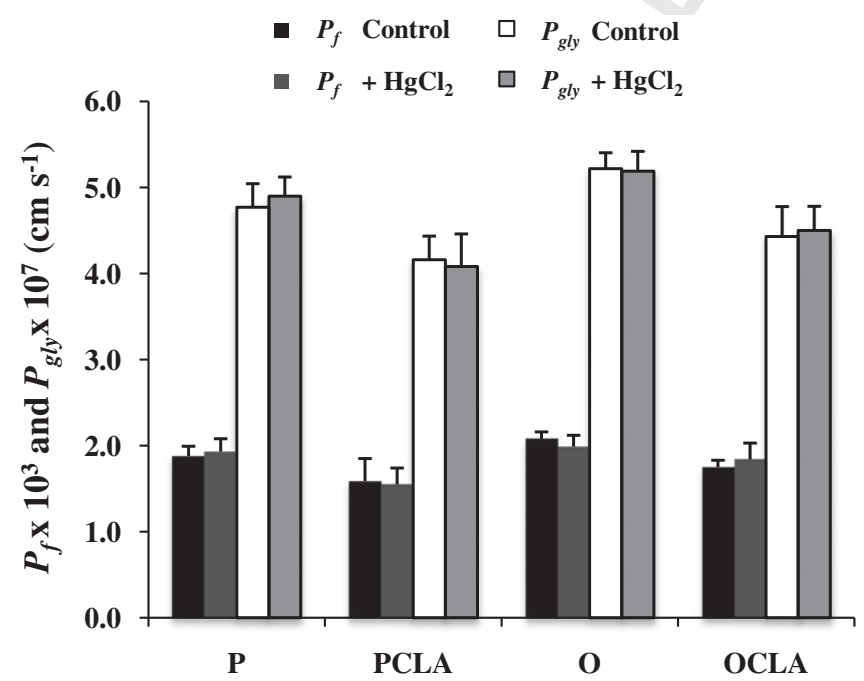

Fig. 4. Permeabilities of water and glycerol at $23^{\circ} \mathrm{C}$ in adipose membrane vesicles from dietary groups $(\mathrm{P}=$ palm oil; $\mathrm{PCLA}=$ palm oil $+1 \%$ conjugated linoleic acid; $\mathrm{O}=$ ovine fat; $\mathrm{OCLA}=$ ovine fat $+1 \%$ conjugated linoleic acid) before and after $\mathrm{HgCl}_{2}$ treatments. Values are mean \pm SEM. No significant effects were detected between control and $\mathrm{HgCl}_{2}$ treatments $(P>0.05)$. shown in Fig. 3. With both fluorescence probes, a significant increase in the viscosity was observed for groups with CLA. Being viscosity inversely related to fluidity, adipose membrane vesicles from dietary groups with CLA are therefore less fluid than without CLA, regardless the fat origin. The membrane rigidification effect associated with CLA supplementation is evident and significant at both membrane depths probed by the two different fluorophores used.

\section{Discussion}

The human health effects of CLA, including the potential to reduce body fat mass, became the subject of widespread research for nearly three decades. Still, its efficiency remains controversial [19]. Depending on the country, estimation of CLA daily intake varies from 15 to $1000 \mathrm{mg}$ [20] thus representing a large range of consumption values. It has been suggested that a consumption of 0.8-3.0 g CLA per day might provide significant health benefits [21], a dosage that in most cases is only achievable through diet supplementation. Besides being the dosage most commonly used in animal research, 1\% CLA is meant to mimic nutritional supplement use.

We thought it should be interesting to look at CLA supplementation effects on adipose membranes obtained from visceral fat of obese Zucker rats. These animals were fed atherogenic and saturated fat diets with distinct origins, vegetable (palm oil) and animal (ovine fat), and the effectiveness of CLA isomers incorporation, the changes of adipose membrane lipid profile and its outcome on fluidity and permeability were assessed in plasma membrane vesicles from visceral adipose tissue. The membrane vesicles obtained herein were homogeneous and enriched in the adipocyte plasma membrane fraction, showed to be osmotically responsive and therefore suitable for transport experiments.

The increase in cholesterol content of a membrane in the liquid disordered state leads to a decrease in its fluidity. On biomembrane 
model systems of certain lipid compositions and on different cell membranes, cholesterol leads to the formation of lipid rafts, microdomains on the liquid ordered state [22] with decreased fluidity that are enriched in cholesterol, sphingolipids and specific proteins [23]. The lipid profile analysis of adipose membranes from the distinct dietary groups has shown that, despite cholesterol content had been influenced by the fat origin, CLA supplementation did not affect cholesterol membrane incorporation. In a similar way, the deposition of SFA, MUFA and PUFA $n-3$ fatty acids were dependent on the fat origin, not presenting any relation to CLA supplementation. As for total PUFA, their total incorporation was not affected by the fat origin or by CLA supplementation. It is known that incorporation of unsaturated fatty acids into cellular membranes increases its fluidity. PUFA acyl chains are extremely flexible and can rapidly change conformational states. The acyl chain flexibility differs substantially between $n-3$ and $n-6$ fatty acids and the number of double bonds significantly alters membrane fluidity. In fact, membrane incorporation of eicosapentaenoic acid EPA $(20: 5 n-3)$ and docosahexaenoic acid DHA $(22: 6 n-3)$, to which numerous health effects have been attributed [24], was significantly dependent on the dietary fat, whereby the effect of CLA was almost negligible. This fact excludes the decrease in DHA or EPA content as responsible for the decrease in adipose membrane fluidity observed in this study, supporting the idea that changes at the fluidity level should be accounted to CLA supplementation rather than to dietary fat. Accordingly, permeability values were consistently lower in adipose membranes when obese Zucker rats were fed CLA, not showing any dependence on the dietary fat origin. Given that the activation energy for both water and glycerol transports was high and relatively stable in all cases, indicating that permeation occurs via the lipid bilayer, the decrease in permeability correlates well with the decrease in membrane fluidity which seems to be caused by CLA incorporation into membrane phospholipids. Despite the same concentration of both CLA isomers in the oil mixture, the $c 9, t 11$, which is the natural occurring isomer in ruminant fat, was detected in a larger amount than the $t 10, c 12$ in all dietary groups, independently of dietary fat. This result per se might suggest that $c 9, t 11$ isomer could be accountable for the changes in the membrane properties reported here. However, given that changes in permeability and fluidity are only observed for CLA supplemented groups and do not depend on the fat origin, the $c 9, t 11$ isomer can be discarded since its concentration is much higher in ovine than in vegetable fat. Therefore, the $t 10, c 12$ isomer may be suggested as the most probable membrane component affecting adipose plasma membrane fluidity and permeability. Interestingly, this CLA isomer is the one with suggested body fat lowering properties in several animal studies [19].

In conclusion, this study suggests that CLA supplementation in obese Zucker rats fed saturated fat and cholesterol rich diets, through its incorporation in membrane phospholipids, has an effect on the fluidity of adipose membranes by increasing membrane rigidity both at the centre of the bilayer (hydrocarbon core) and at a shallow position, closer to the water/lipid interface. This effect could be explained by the geometric structure of the $t 10, c 12$ CLA isomer, where a considerable bending in the fatty acid chain induces a decrease in the average chain length that, similarly to cholesterol, may affect lipid packing within cellular membranes. This isomer and particularly its conformation may promote the formation of other type of raft-like structure, different from the cholesterol and sphingolipids rich microdomains, contributing to the experimentally observed decrease on the average membrane fluidity and permeability of the system. Consequently, our results do not point out to membrane fluidification as a possible mechanism supporting CLA effect as a body reducing agent in obese fat consumers.

\section{Acknowledgments}

This study was supported by FCT grants PTDC/CVT/2006/66114 and PTDC/CVT/2008/99210. We wish thank PharmaNutrients Inc. (Gurnee, IL, USA). We acknowledge Rui Bessa and Susana Alves (L-INIA-REQUIMTE) for fatty acid analysis, Pedro Matos and Teresa Freitas (IMM, FMUL) for assistance in fluidity measurements, and Marta Camps (IRB, Spain) for support in immunoassays.

\section{References}

[1] K.F. Adams, A. Schatzkin, T.B. Harris, V. Kipnis, T. Mouw, R. Ballard-Barbash, A. Hollenbeck, M.F. Leitzmann, Overweight, obesity, and mortality in a large prospective cohort of persons 50-71 years old, N. Engl. J. Med. 355 (2006) 763778.

[2] J.A.M. Prates, R.B. Bessa, Trans and $n-3$ fatty acids, in: L.M.L. Nollet, F. Toldrá (Eds.), Handbook of Muscle Food Analysis, CRC Press, New York, 2009, pp. 399417.

[3] C.J. Field, P.D. Schley, Evidence for potential mechanisms for the effect of conjugated linoleic acid on tumor metabolism and immune function: lessons from $n-3$ fatty acids, Am. J. Clin. Nutr. 79 (2004) 1190S-1198S.

[4] G. Soveral, A.P. Martins, S.V. Martins, P.A. Lopes, C.M. Alfaia, J.A. Prates, T.F. Moura, Effect of dietary conjugated linoleic acid isomers on water and glycerol permeability of kidney membranes, Biochem. Biophys. Res. Commun. 383 (2009) 108-112.

[5] W. Stillwell, S.R. Wassall, Docosahexaenoic acid: membrane properties of a unique fatty acid, Chem. Phys. Lipids 126 (2003) 1-27.

[6] K. Kishida, H. Kuriyama, T. Funahashi, I. Shimomura, S. Kihara, N. Ouchi, M Nishida, H. Nishizawa, M. Matsuda, M. Takahashi, K. Hotta, T. Nakamura, S. Yamashita, Y. Tochino, Y. Matsuzawa, Aquaporin adipose, a putative glycerol channel in adipocytes, J. Biol. Chem. 275 (2000) 20896-20902.

[7] S.V. Martins, P.A. Lopes, C.M. Alfaia, P.O. Rodrigues, S.P. Alves, R.M. Pinto, M.F Castro, R.J. Bessa, J.A. Prates, Serum adipokine profile and fatty acid composition of adipose tissues are affected by conjugated linoleic acid and saturated fat diets in obese Zucker rats, Br. J. Nutr. 103 (2010) 869-878.

[8] M.M. Bradford, A rapid and sensitive method for the quantitation of microgram quantities of protein utilizing the principle of protein-dye binding, Anal. Biochem. 72 (1976) 248-254.

[9] W.W. Christie, G. Dobson, R.O. Adlof, A practical guide to the isolation, analysis and identification of conjugated linoleic acid, Lipids 42 (2007) 1073-1084.

[10] E.D. Naeemi, N. Ahmad, T.K. Al-Sharrah, M. Behbahani, Rapid and simple method for determination of cholesterol in processed food, J. AOAC Int. 78 (1995) 1522-1525.

[11] M.P. van Heeswijk, C.H. van Os, Osmotic water permeabilities of brush border and basolateral membrane vesicles from rat renal cortex and small intestine, J. Membr. Biol. 92 (1986) 183-193.

[12] J.A. Dix, D.A. Ausiello, C.Y. Jung, A.S. Verkman, Target analysis studies of red cell water and urea transport, Biochim. Biophys. Acta 821 (1985) 243-252.

[13] E. Pebay-Peyroula, E.J. Dufourc, A.G. Szabo, Location of diphenyl-hexatriene and trimethylammonium-diphenyl-hexatriene in dipalmitoylphosphatidylcholine bilayers by neutron diffraction, Biophys. Chem. 53 (1994) 45-56.

[14] Z.J. Huang, R.P. Haugland, Partition coefficients of fluorescent probes with phospholipid membranes, Biochem. Biophys. Res. Commun. 181 (1991) 166171.

[15] J.R. Lakowicz, Principles of fluorescence spectroscopy, Kluwer Academic/ Plenum, New York, 1999.

[16] K.V. Kandror, J.M. Stephens, P.F. Pilch, Expression and compartmentalization of caveolin in adipose cells: coordinate regulation with and structural segregation from GLUT4, J. Cell. Biol. 129 (1995) 999-1006.

[17] M.P. Marrades, F.I. Milagro, J.A. Martinez, M.J. Moreno-Aliaga, Differential expression of aquaporin 7 in adipose tissue of lean and obese high fat consumers, Biochem. Biophys. Res. Commun. 339 (2006) 785-789.

[18] R.I. Macey, R.E. Farmer, Inhibition of water and solute permeability in human red cells, Biochim. Biophys. Acta 211 (1970) 104-106.

[19] Y. Park, M.W. Pariza, Mechanisms of body fat modulation by conjugated linoleic acid, Food Res. Int. 40 (2007) 311-323.

[20] S.V. Martins, P.A. Lopes, C.M. Alfaia, V.S. Ribeiro, T.V. Guerreiro, C.M. Fontes, M.F. Castro, G. Soveral, J.A. Prates, Contents of conjugated linoleic acid isomers in ruminant-derived foods and estimation of their contribution to daily intake in Portugal, Br. J. Nutr. (2007) 1-8.

[21] F.C.J. Parrish, B.R. Wiegand, D.C. Beitz, D.U. Ahn, M. Du, A.H. Trenkle, Use of dietary CLA to improve composition and quality of animal-derived foods, in: J.L. Sébédio, W.W. Christie, R. Adlof (Eds.), Advances in Conjugated Linoleic Acid Research, AOCS Press, Champaign, IL, 2003, pp. 189-217.

[22] D. Marsh, Cholesterol-induced fluid membrane domains: a compendium of lipid-raft ternary phase diagrams, Biochim. Biophys. Acta 1788 (2009) 21142123.

[23] D. Lingwood, K. Simons, Lipid rafts as a membrane-organizing principle, Science 327 (2010) 46-50.

[24] J.J. Li, C.J. Huang, D. Xie, Anti-obesity effects of conjugated linoleic acid, docosahexaenoic acid, and eicosapentaenoic acid, Mol. Nutr. Food Res. 52 (2008) 631-645. 